\title{
Préserver pour la postérité : des anciens de l'ethnie bundjalung au musée national d'ethnologie des
} Pays-Bas

\section{Fanny Wonu Veys}

\section{(2) OpenEdition Journals}

Édition électronique

URL : http://journals.openedition.org/jso/6919

DOI : $10.4000 /$ jso.6919

ISSN : $1760-7256$

Éditeur

Société des océanistes

\section{Édition imprimée}

Date de publication : 15 octobre 2013

Pagination : 63-76

ISBN : 978-2-85430-035-2

ISSN : 0300-953x

Référence électronique

Fanny Wonu Veys, «Préserver pour la postérité : des anciens de l'ethnie bundjalung au musée national d'ethnologie des Pays-Bas », Journal de la Société des Océanistes [En ligne], 136-137 | 2013, mis en ligne le 14 novembre 2013, consulté le 21 avril 2020. URL : http://journals.openedition.org/jso/6919 ; DOI : https://doi.org/10.4000/jso.6919 


\section{Préserver pour la postérité : des anciens de l'ethnie bundjalung au musée national d'ethnologie des Pays-Bas ${ }^{1}$}

par

Wonu VEYS

\section{RÉSUMÉ}

Le 29 septembre 2009, deux anciens de l'ethnie bundjalung sont venus à Leyde pour le rapatriement officiel de restes humains, dits "ancêtres", collectés en 1882 dans la région de la Richmond River par A. P. Goodwin et transférés $d u$ musée aux collections anatomiques $d u$ centre universitaire médical de Leyde (LUMC). Le rapatriement était pour les Bundjalung un acte politique pouvant inspirer et encourager d'autres pays ou musées à faire de même. Toutefois, ce qui nous importe ici, c'est la visite des objets collectés par A.P. Goodwin dans la même région et à l'époque de l'acquisition des ossements. Il est impossible de comprendre la signification actuelle des objets sans examiner la portée des pratiques de collection et des vicissitudes liées à la colonisation du territoire bundjalung à la fin du XIX siècle. La visite a démontré que ces objets, devenus obsolètes aujourd'hui, ont le pouvoir, par leur présence, de provoquer la mémoire, révélant ainsi leur part immatérielle. De là à engager une réflexion plus ample sur le rôle médiateur présent et futur des musées ethnographiques, il n'y a qu'un pas.

Mots-ClÉs : Aborigènes d'Australie, culture matérielle, pratiques de collection, rapatriement, musée d'ethnographie

\begin{abstract}
On 29 September 2009, two elders from the Bundjalung people came to Leiden for the official repatriation of human remains, referred to as ancestors, that were collected in 1882 in the Richmond River area by A. P. Goodwin and transferred from the museum to the anatomical collections of the Leiden University Medical Centre (LUMC). This repatriation was for the Bundjalung people a political action, which could inspire and encourage other countries or museums to do the same thing. However, the focus of this article is the viewing of the objects that were collected by $A$. P. Goodwin at the same time and place as the human remains. It is impossible to understand the current meaning of these objects without examining collection practices and the vicissitudes connected to the colonisation of the Bundjalung territory at the end of the nineteenth century. The visit to the museum stores has shown that these objects, now obsolete, have the power through their presence, to provoke memory, thus revealing their part of immateriality. Engaging with a larger reflection on the mediating role of present and future ethnographic museums is a small step from here.
\end{abstract}

KeYwords: Australian Aborigine, material culture, collection practices, repatriation, ethnographic museum

1. Je tiens à présenter toutes mes excuses quant aux informations émises dans cet article. En effet, les Aborigènes d'Australie et plus spécifiquement le peuple bundjalung peuvent les ressentir comme pénibles. Ces données sont pourtant essentielles à ce récit.

*Rijksmuseum Volkenkunde, Leyde (Pays-Bas), wonu.veys@volkenkunde.nl 
Le 29 septembre 2009, deux anciens du peuple bundjalung faisaient la une des journaux hollandais et australiens. Ils étaient venus aux PaysBas pour le rapatriement officiel des ossements humains, collectés en 1882 par Adolphe Peter Goodwin avec l'aide de Jürgen Heinrich Witt, dans la région de la Richmond River, restes qui donc appartenaient aux ancêtres des deux anciens Bundjalung. Comme ce «retour à la maison " de restes d'ancêtres était une première pour les Bundjalung, les anciens avaient le sentiment d'écrire un bout d'Histoire. Il importait aux gouvernement et ambassade d'Australie, au gouvernement des Pays-Bas ainsi qu'aux anciens Bundjalung que cet événement connaisse une publicité aussi vaste que possible. Le rapatriement était pour les Bundjalung un acte politique pouvant inspirer et encourager d'autres pays ou musées à faire de même. Toutefois, ce qui nous occupe ici, c'est la rencontre avec des objets collectés par A. P. Goodwin dans la région et à l'époque où furent acquis les ossements susmentionnés. Il est impossible de comprendre la signification actuelle des objets sans examiner la portée des pratiques de collection et des vicissitudes liées à la colonisation du territoire bundjalung à la fin du XIX ${ }^{e}$ siècle. La visite a démontré que ces objets, devenus obsolètes aujourd'hui, ont le pouvoir, par leur présence, de provoquer la mémoire, révélant ainsi leur part immatérielle. De là à engager une réflexion plus ample sur le rôle médiateur présent et futur des musées ethnographiques, il n'y a qu'un pas.

\section{Rapatriement}

La visite et le rapatriement officiel doivent être lus sur fond de voix s'élevant avec une insistance toujours accrue dans les dernières décennies du $\mathrm{xx}^{\mathrm{e}}$ siècle et défiant « le droit des musées de raconter leurs histoires [celles des peuples aborigènes] et de garder des collections d'objets obtenues de leurs ancêtres [aborigènes]" (Nicks, 2003 : 19). En outre, les objets matériels ont le pouvoir d'incarner le sens de la tradition. Ils aident un groupe spécifique à légitimer les revendications d'une identité unique et d'un pouvoir politique dans la société ambiante (Kaplan, 2011 : 153).

À propos de rapatriement d'objets et de restes humains aborigènes, certains moments clés retiennent notre attention. Ces vingt dernières années, des Aborigènes ont découvert dans des musées australiens les restes d'au moins 7200 de leurs ancêtres ; 5500 d'entre eux ont une provenance connue (Cubillo, 2010 : 20). En 1978, des Australiens aborigènes au séminaire régional de l'UNESCO exprimèrent avec vigueur leur désir d'accéder aux collections publiques de culture matérielle aborigène australienne en Australie et mirent l'accent sur le rôle vital des propriétaires et représentants de communautés aborigènes dans le réveil des collections d'objets (Bolton, 2003 : 44). Paul Turnbull (2010 : 117) explique qu'à partir des années 1980 les efforts des Aborigènes d'Australie pour récupérer des ossements et autres restes humains ont reçu une écoute considérable. En entendant les revendications aborigènes, nombre de chercheurs éminents en archéologie, anatomie et anthropologie biologique ont même fini par se poser des questions quant à la pertinence de l'appropriation de ces restes humains. En dépit de la résistance de l'opinion publique et de certains autres chercheurs, des requêtes furent introduites. En 1982, le Tasmanian Aboriginal Centre à Hobart contacta l'Université d'Édimbourg pour le retour du crâne du chef aborigène William Lanne, alias King Billy, ainsi que de dix autres crânes d'Aborigènes de Tasmanie. Cette collection anatomique appartenait à une des plus grandes collections rassemblées hors des frontières australiennes. Autrefois, Sir William Turner, professeur d'anatomie à Édimbourg de 1867 à 1903 et collectionneur de restes humains avait souligné l'importance de garder réunis les crânes de différentes ethnies. Cependant, les restes de William Lanne furent rendus en 1991 accompagnés d'une vaste collection d'autres restes humains parmi lesquels ceux de quatre-vingt-sept individus restitués à la communauté Larrakia à Darwin et ceux de trois cents ancêtres à la communauté Ngagarrindjeri (Cubillo, 2010 : 23). D'autres musées suivirent cet exemple dont celui de la Bradford University, le Glasgow Museum, le Peterborough City Museum et le Pitt Rivers Museum d'Oxford.

En 1983, des Aborigènes d'Australie proposèrent de considérer les restes humains comme propriété religieuse ce qui impliqua le retrait des restes de la sphère publique pour les garder dans des endroits spéciaux et donc moins accessibles à un public non aborigène (Greenfield, 2007 : 302). En même temps, le repérage des ossements dans des institutions publiques demeurait aussi important qu'avant. Il fut établi que plusieurs musées en Europe possédaient des restes humains. L'Angleterre, l'Ecosse, la Belgique et l'Autriche s'opposèrent à leur retour tandis que les États-Unis, la Suède et la France étaient partisans de préserver ces restes humains dans un emplacement particulier. En 1989, le World Archaeological Congress adopta le Vermillion Accord sur les restes humains en faveur des droits des peuples indigènes à la restitution des restes de leurs ancêtres, reconnaissant implicitement que leur retour était important pour la survie et la vitalité des cultures indigènes. Turnbull avance que l'accord marque " un changement considérable dans la compréhension du rapatriement au sein des cercles scientifiques " (Turnbull, 2010 : 118). En 1990, il devint évident que l'acqui- 
sition de crânes et de squelettes avait souvent été le résultat de meurtres perpétrés sur des Aborigènes (Turnbull, 2010 : 122). Le pillage des tombes afin d'en récupérer les ossements fut aussi une pratique courante. En 2002, des restes humains comprenant probablement ceux de Trugernanner, souvent appelée Truganini, qui selon la légende populaire fut considérée comme la dernière femme aborigène de Tasmanie et qui mourut en 1876, furent rendus par le Royal College of Physicians de Londres. Ses cendres furent dispersées sur la côte méridionale de la Tasmanie. En 2003, le retour des crânes aborigènes qui avaient été exposés pendant plus de cent ans au Museum of Manchester fut une réalité. Ils furent rapatriés à Victoria et sont gardés maintenant en un lieu sacré dans l'Australian Capital Territory. Le Royal College of Surgeons et le Horniman Museum à Londres ont également restitué des crânes. Le Bristol City Museum souhaitait aussi remettre des restes humains en 2004. Au courant de cette même année quelques discussions supplémentaires sur le rapatriement ont vu le jour. L'une d'entre elles a eu lieu au Leverhulme Centre for Evolutionary Studies à Cambridge, qui abrite une collection de 18000 ossements et crânes connue sous le nom de la collection Duckworth. L'argument avancé était que la collection devait rester sur place afin de pouvoir effectuer des recherches. Les représentants aborigènes ont répliqué que cet argument était fallacieux, car leurs ancêtres n'avaient pas vu le jour afin d'être des objets de recherche. Une façon de penser similaire existait à propos de la collection de restes humains au Natural History Museum. Là, l'opinion était que 10000 restes humains non britanniques soient considérés comme matériel de recherche et non comme des ancêtres d'un peuple vivant (Greenfield, 2007 : 304-305). Quelques restitutions comme celle d'Ann Arbor dans le Michigan et celle de Suède ont été couronnées de succès en 2004. L'histoire de la collection se trouvant en Suède a été révélée lors de la publication du journal d'Eric Mjöberg. Il était clair que les objets et les restes humains avaient été obtenus illégalement. Pour la première fois, un pays autorisait le rapatriement inconditionnel en faveur du gouvernement australien (Hallgren, 2010 : 135-143). Selon Paul Turnbull, depuis le Vermillion Accord:

« une appréciation historique plus éclairée du comment et du pourquoi de l'obtention et de l'utilisation de restes humains à des fins scientifiques montre la distance cognitive entre les chercheurs contemporains et les hypothèses et pratiques des scientifiques d'antan impliqués dans la profanation de cimetières autochtones. » (2010: 131)

\section{Le rapatriement de Leyde}

Le Rijksmuseum Volkenkunde (RMv) - le musée national d'ethnologie - à Leyde a pris la position de médiateur dans le rapatriement de 2009 parce qu'une partie des restes humains avait appartenu aux collections muséales. Ces ossements avaient été transférés vers la collection anatomique du Leids Universitair Medisch Centrum (LUMC) - le Centre médical universitaire de Leyde - en 1935 en provenance du RMV. Au LUMC, il y a peu de données et il est impossible de savoir exactement de combien d'ossements il s'agissait. Les plus anciennes fiches individuelles des restes humains au LuMC datent de 1977. Il faut donc consulter les archives du RMV pour en apprendre plus. Le 27 mars 1882, le RMV avait acheté de J. H. Witt à Hambourg en Allemagne trois restes d'Aborigènes australiens appartenant à deux individus. Le récépissé et le journal de collection du musée attestent de l'origine des ossements qui viennent de la région de la Richmond River dans la Nouvelle-Galles du Sud. La collection RMV mentionne en outre que les restes ont été remis à J. H. Witt par A. P. Goodwin. Par la même occasion, le RMv a acheté d'autres objets Goodwin : douze boomerangs, quatre boucliers, six lances, quatre massues, un burrawang (une graine de cycadale), trois filets de pêche, douze sacs en vannerie, tous avec mention de provenance australienne. Une des lances a été échangée avec un autre musée, un sac et la graine n'ont jamais été enregistrés et deux autres sacs ont été attribués à une date ultérieure à l'île de Pâques (Rapanui) et à Tahiti. Il y avait également une hache en pierre originaire, selon Goodwin, des îles Salomon (RMv 295-58) ${ }^{2}$.

Le 29 septembre 2009, les ossements de trois autres Aborigènes d'Australie ont également été restitués par le Lumc. Personne ne sait d'où en Australie ces restes humains proviennent. Un crâne a été identifié comme étant issu de la Nouvelle-Galles du Sud mais au moment de la collecte ce nom désignait toute la côte Est de l'Australie. Ce crâne a été présenté initialement au professeur de l'université de Leyde, Sebald Brugmans, par le Britannique Sir Joseph Banks, spécialiste en histoire naturelle et botaniste, président de la Royal Society, probablement en 1794. Si c'est le cas, il s'agit d'un crâne assez ancien puisqu'à cette époque il n'y avait que six ans que la Grande-Bretagne avait colonisé la Nouvelle-Galles du Sud et fondé Sydney. Suite à la mort de Brugmans en 1819, les ossements ont été transférés à la collection d'anatomie de l'université de Leyde. Les deux autres crânes sont des crânes qui en 1977 ont été répertoriés par le 


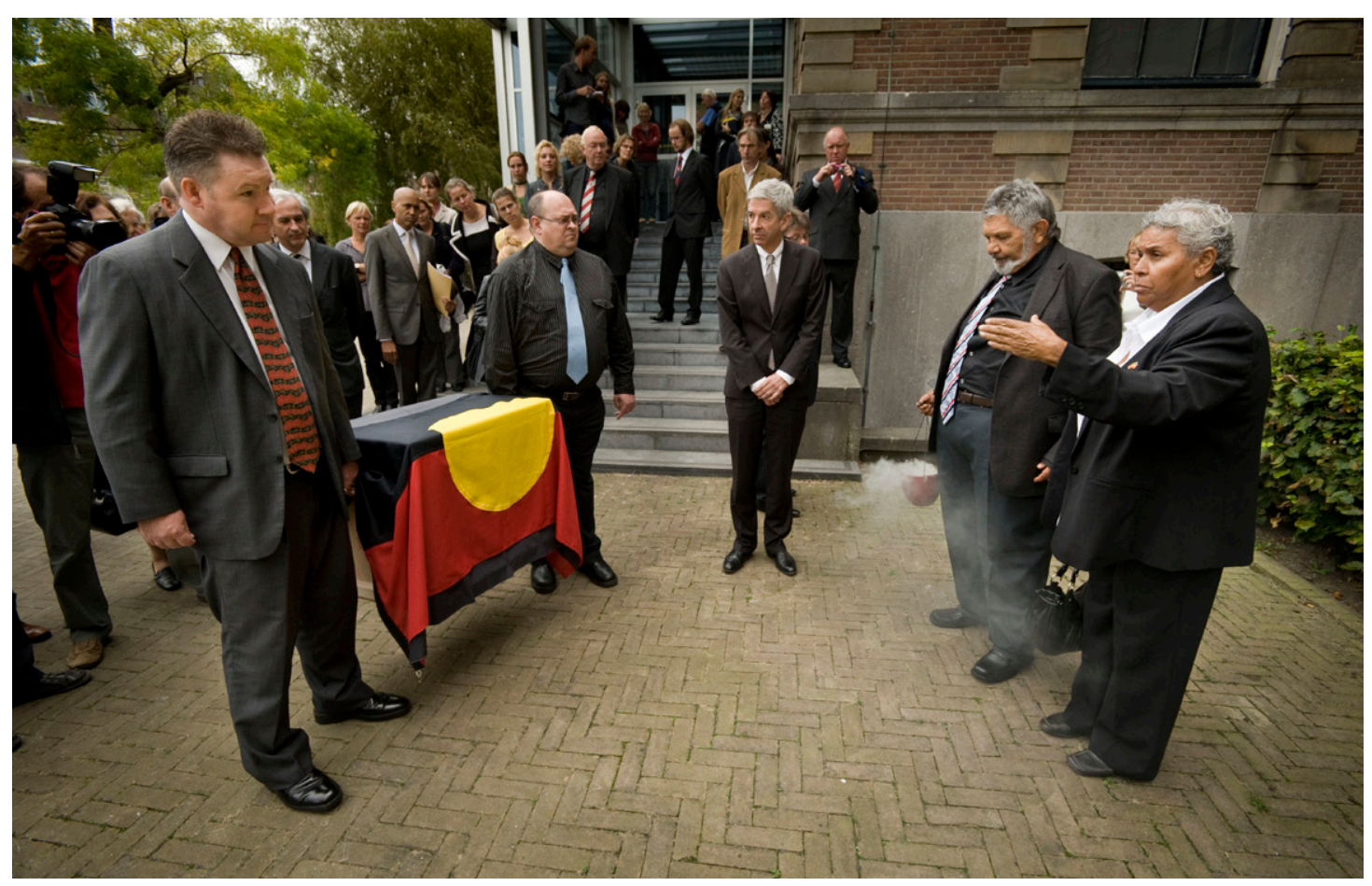

Рното 1. - Cérémonie de fumigation (@ Taco van der Eb, 29/09/2009, Leyde)

LUMC comme étant probablement d'origine australienne. Une analyse morphologique ultérieure a démontré que ces crânes avaient effectivement une origine australienne autochtone.

Le programme international de rapatriement (International Repatriation Program) qui est organisé par le Department of Families, Housing, Community Services and Indigenous Affairs (FaHCSIA), a initié la relation entre les propriétaires légaux, la Collection anatomique au LUMC et le gouvernement australien agissant pour les anciens Bundjalung. Le ministre hollandais de l'Éducation, de la Culture et de la Science de l'époque, Ronald Plasterk, était présent lors du transfert officiel. Deux cercueils couverts du drapeau aborigène rouge, noir et jaune ont été remis. L'un contenait les restes clairement identifiés comme provenant de la contrée de la Richmond River; l'autre renfermait des restes aborigènes non identifiés. C'est toujours un défi de savoir quoi faire avec des restes humains non documentés et sans affiliation connue (Rosoff, 2003 : 74). En effet, les restes appartenant aux trois individus non identifiés témoignent de cette difficulté puisqu'ils se trouvent toujours à l'Australian National Museum qui fait fonction de dépositaire temporaire dans ces cas-là (Byers, 2013). L'ambassadeur australien de l'époque aux Pays-Bas, Lydia Morton, a souligné l'importance de ce retour, parce qu'il pourrait aider à " réparer les injustices dont le peuple aborigène australien a pu souffrir ». Tout en paraphrasant la politique officielle du gouvernement australien, elle y ajoutait aussi un aspect de responsabilité éthique :

«Le gouvernement australien reconnaît que la préservation, le soutien et la promotion de la culture, de l'art et de l'héritage autochtones sont des composants essentiels face à certains désavantages auxquels les autochtones australiens ont été soumis. Le gouvernement reconnaît également que la culture, l'art et l'héritage autochtones de l'Australie sont des forces puissantes et dynamiques dans la société autochtone contemporaine et forment un aspect unique de la vie australienne." (Australian Federal Department of Foreign Affairs and Trade, in Morton, 2010 : 96)

Mme Morton a ainsi suggéré que le rapatriement n'est pas seulement une question nationale et une manière de réattribuer du pouvoir aux Australiens autochtones, mais aussi une occasion pour tous les Australiens de reconnaître que l'Australie n'était pas une terra nullius jusqu'à l'arrivée des colons blancs. Pour la Bundjalung Gwenda Hickling, une ancienne, l'événement faisait partie du processus de réconciliation, rendant à son peuple ce qui lui avait été enlevé ( $R a-$ dio Netherlands Worldwide 2009).

\section{Cérémonies}

Henry Atkinson (2010 : 19), porte-parole du peuple Yorta Yorta dont le territoire est situé dans la contrée de Murray-Goulburn dans la 
Nouvelle-Galles du Sud et l'État de Victoria a lancé un plaidoyer s'adressant aux « hommes et femmes du monde scientifique»:

« Les ossements des nôtres doivent recevoir les honneurs coutumiers avant de quitter la prison qui les a retenus pendant tant d'années. Les dépositaires autochtones donneront aux nôtres le respect qui leur est dû et les ramèneront chez eux. Les autochtones doivent pouvoir vivre cette connexion spirituelle avec leurs ancêtres. Cela débute avec des cérémonies rituelles présidées par les dépositaires autochtones. Les restes sont libérés de leur lieu d'enfermement obscène avant qu'ils ne commencent leur long voyage de retour. Chez eux ils seront rejoints par la communauté autochtone qui les attend et après des cérémonies traditionnelles de rigueur, ils seront remis à la terre de leur début. » (Atkinson, 2010 : 19)

Dans cet esprit, le Rijksmuseum Volkenkunde a répondu aux souhaits des deux anciens qui représentaient la communauté bundjalung, c'està-dire Gwenda Hickling et Desmond 'John' Morrissey qui ont tenu une cérémonie de fumigation en brûlant des feuilles vertes d'eucalyptus de manière à obtenir de la fumée. La fumée a été dirigée sur les cercueils et les personnes intimement liées à la procédure de rapatriement. Le but était de débarrasser celles-ci de toute charge négative tels les soucis et les angoisses, permettant un renouveau par purification. Ensuite, ils ont expliqué ce qu'ils venaient de faire. Pour Gwenda Hickling, c'était un moment très important. Dans une interview pour la Radio Netherlands Worldwide elle a exprimé les choses comme suit :

"C'est spirituel. Nous vivions dans le Rêve. Nous venons du Rêve. La terre est notre Rêve. Ceci est une part du Rêve qui revient chez lui. "

C'était la première fois que ces anciens prenaient part à pareil rapatriement cérémoniel. Ils nous ont raconté qu'ils avaient essayé de trouver des voies fidèles à leur communauté tout en respectant leurs croyances chrétiennes. Jusqu'en 2010, les ossements bundjalung étaient entreposés au National Museum of Australia. Entretemps, il y a eu des consultations concernant leur retour en terre natale entre le conseil des anciens, c'est-à-dire le Bundjalung Elders Council Aboriginal Corporation dans la Nouvelle-Galles du Sud et le NSW Department of Environment and Climate Change (Parcs nationaux) (Byers, 2010 ; Byers, 2013). Le National Museum of Australia a pris son rôle au sérieux pour aider à l'organisation de l'enterrement des ancêtres. Ce musée définit son rôle comme suit :

« Le musée conseille et assiste les institutions d'héritage culturel fédérales, celles des États et des Territoires, les communautés autochtones et leurs représentants pour le rapatriement de restes humains autochtones ainsi que d'objets secrets/sacrés. Le musée procure également des informations aux médias et au public en général sur le rapatriement. La gestion des restes humains et des objets secrets/sacrés est strictement contrôlée par la section de rapatriement du $\mathrm{Mu}$ sée afin d'assurer un soin culturellement sensible et approprié au matériel en accord avec les meilleures pratiques muséales. » (National Museum of Australia 2008-2009)

Lenterrement des deux ancêtres a finalement eu lieu le 26 novembre 2010 en présence de Noel James, Hazel Rhodes, Arthur Ferguson, Bertha Kapeen, Dorothy Harris-Gordon, John Morrissey et Gwenda Hickling. Ces deux derniers ont donc pu suivre tout le trajet, depuis le rapatriement jusqu'à l'enterrement. Les restes, enveloppés dans de l'écorce, ont été placés sur une table couverte du drapeau aborigène, après quoi des étudiants de la Goonellabah Public School ont entonné un chant de bienvenue. Les deux ancêtres ont été bénis par Dorothy Harris-Gordon, une ancienne (Buchanan, 2010).

\section{L'histoire des objets}

Le jour suivant la cérémonie de rapatriement, les deux anciens de l'ethnie bundjalung sont venus voir les objets dans les réserves externes du Rijksmuseum Volkenkunde. Comme mentionné ci-dessus, quarante-deux objets avaient initialement été collectés à la même période que les ossements bundjalung ancestraux, dans la même région, par le même homme, A. P. Goodwin. C'est cet aspect plus caché de leur visite qui nous importe à présent. Tandis qu'un comité éthique s'était prononcé sur le pour et le contre d'un rapatriement des restes humains et sur la nature des considérations dont il fallait tenir compte, les objets, eux, ne jouaient aucun rôle dans l'histoire. C'est pourtant un fait bien documenté qu'ils cristallisent pour les Aborigènes des questions sensibles et essentielles. C'était le cas pour trois peintures sur écorce que le Museum Victoria avait empruntées en 2004 du Royal Botanic Gardens à Kew et du British Museum pour faire partie d'une exposition sur l'héritage culturel et spirituel des Dja Dja Wurrung. Elles étaient pour eux la seule preuve de leur existence en tant que clan et de leur attachement à une terre particulière. À l'origine, ces objets avaient été collectés dans les années 1850 par John Hunter Kerr, un propriétaire terrien de Loddon River né à Édimbourg en Écosse en 1821. En 2005, les anciens du peuple Dja Dja Wurrung ont cherché à obtenir une décision judiciaire fédérale pour empêcher le retour en Angleterre des peintures d'écorce de Victoria, leur lieu d'origine. Au début, il semblait que le peuple 
Dja Dja Wurung allait obtenir gain de cause, mais, après beaucoup de discussions légales et un jugement par la Cour fédérale d'Australie, les objets ont été remis aux deux musées britanniques (Greenfield, 2007 : 304-310). Mon argument est que la rencontre avec les objets au RMV a eu un impact probablement plus profond sur les anciens bundjalung que le retour des restes humains, ce qui est révélateur de l'importance émotionnelle d'objets matériels.

\section{Le collectionneur Adolph Peter Goodwin}

Le collectionneur Adolph Peter Goodwin (1842-1914) était un naturaliste, collectionneur, taxidermiste, photographe et marchand né au Danemark. Sa collection d'Australie comportait trente-sept artéfacts ${ }^{3}$. Outre sa date de naissance, les premières données de sa vie font référence à son arrivée en Australie en 1876 après avoir passé quelques temps en Californie. Sa rubrique nécrologique dans le Northern Star (1914) raconte que A. P. Goodwin avait reçu une formation de naturaliste dès son plus jeune âge et que sa recherche continuelle de connaissances nouvelles sur la faune et la flore l'amena à entreprendre trois à quatre [sic] voyages autour du monde. En 1887, il acheta une propriété à Lismore, en plein estuaire de la rivière Richmond, dans le nord de la Nouvelle-Galles du Sud en Australie (The Northern Star, 1887), où il travailla pendant quelques temps comme photographe et collectionneur d'objets d'histoire naturelle. En 1889, Goodwin se trouvait en Papouasie NouvelleGuinée en train de faire une collecte ornithologique quand il rencontra J. B. Cameron à Port Moresby. Ce dernier faisait partie de l'expédition de Sir William MacGregor qui explorait la chaine montagneuse Owen Stanley en Papouasie Nouvelle-Guinée. Alors que l'expédition était déjà dans la région de la Vanapa River, Goodwin fut embauché comme nouveau membre. Il profita de l'occasion pour collecter des spécimens d'histoire naturelle, mais aussi pour réaliser des plaques de lanterne magique (Goodwin, 1890 : 150; Northern Star, 1914). À son retour de sa courte expédition en Nouvelle-Guinée, Goodwin voyagea jusqu'à Londres en Angleterre via Cooktown dans le Nord Queensland. À Londres, ses plaques de lanterne magique ${ }^{4} \mathrm{ac}-$ compagnaient le rapport que Sir W. Macgregor présenta le 9 décembre 1889 à la Royal Geographic Society de Londres et qui était intitulé
" Journey to the Summit of the Owen Stanley Range, New Guinea "(Duff, 1890). Goodwin fut élu membre de la Royal Geographical Society tandis que la Linnean Society l'aida à publier ses Notes on the Paradise Birds of British New Guinea (Goodwin, 1890). À son retour d'Angleterre, il séjourna brièvement à Perth avant de partir pour Lismore en 1890 où son fils Fred Goodwin travaillait pour le journal le Northern Star comme membre du personnel littéraire. Adolph Goodwin acheta une propriété dans la Zadoc Street, fit construire un magasin et une maison, et poursuivit ses activités de taxidermiste jusqu'aux alentours de 1903 lorsqu'il déménagea à Tallow Beach, à quelque cinq kilomètres de Byron Bay dans la Nouvelle-Galles du Sud. À partir de 1895, on rapporte que Goodwin a des difficultés financières. Le 19 avril, B.C. Wood \& Co organisa pour lui une vente aux enchères d'oiseaux empaillés, de spécimens d'histoire naturelle et de mobilier à sa maison à Perth - où il avait passé quelques mois en 1890 - sur l'angle de Lake Street et Hardinge Street (The West Australian, 1895). Il s'agissait de plus de 125 variétés d'oiseaux empaillés, d'accessoires photographiques et d'une lanterne magique fabriquée par Hughes et Parfingas, des plaques faites lors de son expédition en Nouvelle-Guinée et des livres tels que Aborigines of Victoria de Robert Brough Smyth (1878), History of British Birds de F. O. Morris (1851-1857) et le Picturesque Atlas of Australasia (1886) (The Daily News, 1895). En 1896, il découvrit une embarcation inconnue rejetée sur le rivage de Byron Bay dont il fit mention à ses amis de la Royal Geographical Society à Londres (Goodwin, 1896). Lors de la mort de sa cousine, Mademoiselle Clausen, en 1908, Goodwin voulut disposer de sa propre collection et créer un musée à Lismore. Ses tentatives furent vaines, ce qui le força à vendre sa collection à des acheteurs privés. Goodwin mourut à Sydney le 18 septembre 1914 à l'âge de 72 ans après avoir souffert d'ataxie locomotrice, qui entravait grandement ses mouvements. Goodwin avait photographié et collecté des armes, des masques et des oiseaux en Papouasie Nouvelle-Guinée et les vendit à des musées et à des collectionneurs (The Northern Star, 1914).

Entre 1883 et 1890, Goodwin vendit 561 objets au RMv de Leyde. S'il vendit certains objets provenant d'Afrique et des Amériques (Nord et Sud), la plus grande partie de sa collection était océanienne. Il est possible qu'une partie de ses objets australiens ait été acquise par le musée Godeffroy ou par des collectionneurs à Bowen

3. Le compte initial du nombre d'objets était de quarante-deux, mais comme expliqué plus tôt, un objet fut échangé, deux autres ne furent jamais enregistrés et à deux objets furent attribués une autre provenance.

4. Au moins une des plaques de A. P. Goodwin est à la Royal Geographical Society à Londres (S0023173). Elle fut prise le 17 juin 1889 et la légende complète est la suivante : "Mount Oven Stanley, New Guinea (Distant view). Photographed from Mount Musgrave at an altitude of 9,000 feet, June 17th, 1889, on Sir William McGregor's, K.C.M.G., Expedition. Distant 7 miles. By A.P. Goodwin». 
et Rockhampton où J. H. Witt doit s'être rendu en qualité de capitaine naviguant pour la compagnie Godeffroy. Nous savons que J. H. Witt faisait activement la collecte et le commerce d'objets avec des données de provenance tellement précises qu'elles en étaient pénibles à lire (Schmeltz, 1899 : 121-124). Lautre matériel de Goodwin en provenance du Pacifique venait de la Papouasie Nouvelle-Guinée (Port Moresby), des îles d'Entrecasteaux, des îles Trobriand, du Vanuatu, de Fidji, des îles Salomon et de la Nouvelle-Bretagne. Il est intéressant de noter que Goodwin a acquis la plupart du matériel de Papouasie Nouvelle-Guinée six ans avant de rejoindre, en 1889, l'expédition William MacGregor. Goodwin, comme beaucoup de ses contemporains, collectait des objets, probablement avec la conviction qu'ils représentaient un peuple et rendaient compte d'une façon de vivre qui était appelée à disparaitre dans un avenir proche. Sa collection nous montre donc les objets qu'il considérait comme précieux à l'époque où il vivait, c'est-à-dire au XIX ${ }^{\mathrm{e}}$ siècle (Clifford, 1988 : 221).

Goodwin a probablement collaboré avec le musée Godeffroy pour le matériel australien en travaillant selon les règles et habitudes de collecte de musée en vigueur au XIx ${ }^{\mathrm{e}}$ siècle. En ce qui concerne les restes humains aborigènes, ainsi que l'explique le Dr. George Dorsey (Dorsey, 1896), Goodwin suivit la tendance de ses contemporains, colons et voyageurs en Australie au XIX ${ }^{e}$ siècle, de ne pas respecter le caractère sacré des tombes. Il semble que A. P. Goodwin se lia d'amitié avec des Aborigènes afin d'en apprendre davantage sur les lieux de sépulture en vue de l'obtention de squelettes. Sa correspondance de mars 1891, conservée au Peabody Museum, est éloquente. Goodwin y explique qu'il connaissait personnellement Neddy Larkin qui mourut de tuberculose, qu'il vit que ce dernier fut enterré en lisière du cimetière de Lismore et qu'il se procura personnellement les restes de cet homme bundjalung5. Enfin, il vendit au Peabody $\mathrm{Mu}-$ seum la photo de Larkin prise avant sa mort pour permettre la comparaison de celle-ci avec le crâne et le squelette du Bundjalung (Dorsey, 1896 ; Turnbull, 1997).

\section{La région de la Richmond River}

Les objets conservés au Rijksmuseum Volkenkunde à Leyde ont été collectés dans la région de la Richmond River, située en territoire bundja-

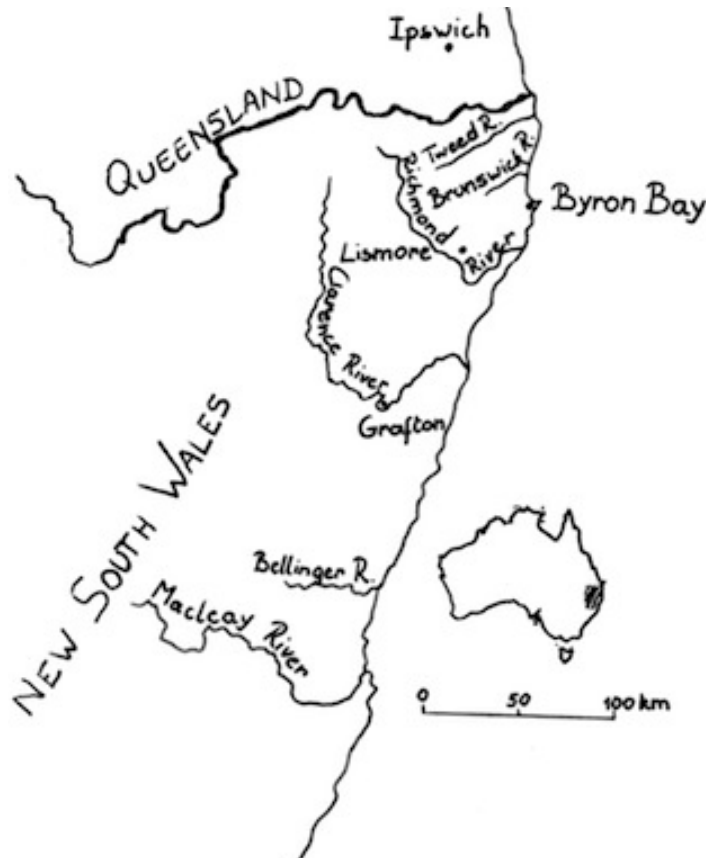

CARTE 1. - Région de la Richmond River (C) Fanny Wonu Veys)

lung. Les membres de cette ethnie sont les gardiens des régions côtières du nord-est de l'Australie, quelque $750 \mathrm{~km}$ au nord-est de Sydney. Les Bundjalung forment en fait une large nation aborigène qui constitue une fédération comprenant un nombre de groupes ou de clans qui occupent le territoire allant de Grafton et de la rivière Clarence dans le nord de la Nouvelle-Galles du Sud aux villes d'Ipswich et de Beaudesert au sud du Queensland. Le territoire s'étend vers l'autre côté de la Cordillère australienne (Great Dividing Range) pour rejoindre Grafton. Les Bundjalung sont entourés par les nations Yuggera et Barrunggam au nord, la mer de Tasmanie à l'est, la nation Gumbaiggir au sud et Ngarabal à l'ouest. La région compte parmi les plus riches terrains de chasse et de pêche du continent australien (Horton, 1994 : 163-164) ${ }^{6}$. Elle entretenait par conséquent une population relativement importante de chasseurs-cueilleurs. Le capitaine James Cook aperçut une vingtaine de Bundjalung le 15 mai 1770 . Le contact avec l'ouest de l'Australie ne commença réellement qu'en 1828 lorsque Henry John Rous, à bord de la frégate HMS Rainbow, jeta l'ancre à Byron Bay avec mission de découvrir une rivière navigable et un lieu d'ancrage sûr. Tandis que Rous " découvrit " la rivière Richmond en août 1828 , ce n'est qu'en 1842 que les premiers coupeurs de cèdres arrivèrent dans le district de la Richmond River en

5. George Dorsey (1896) emploie Paikalyug en se référant aux Bundjalung.

6. Selon les traditions orales du peuple bundjalung, ces contrées étaient habitées par trois frères et leurs descendants. Les noms des quinze groupes tribaux formant la nation bundjalung sont Aragwal, Banbai, Birbai, Galiabal, Gidabal, Gumbainggeri, Jigara, Jugambal, Jugumbir, Jungai, Minjungbal, Ngacu, Ngamba, Thungutti et Widjabal. 
quête de magnifiques arbres (Daley, 1966: 11, 27). Ce fut le premier contact permanent entre les colons et les Aborigènes de la Richmond River (à ne pas confondre avec les Bundjalung près de Clarence). Des fermiers et des éleveurs de la colonie de Port Jackson rejoignirent la région, obligeant la population aborigène à se retirer sur le contrefort des montagnes, ce qui perturba la culture et l'économie tribales. Les Bundjalung sont donc entrés en contact avec les colons très tôt dans l'histoire coloniale de l'Australie. Dès les années 1880, il ne subsistait que peu d'Aborigènes bundjalung vivant dans les petits camps dans les collines. Ils travaillaient comme gardiens de bestiaux ou coupeurs de bois dans les stations. Avant même la possibilité d'une étude anthropologique méticuleuse et de l'enregistrement de données ethnographiques, cette culture aborigène fut sérieusement perturbée (McBryde, 1978 : 135 ; Sullivan, 1978 : 101-102). Ce sentiment de perte de savoir a été exprimé à plusieurs reprises par Gwenda Hickling et Desmond 'John' Morrissey lors de la rencontre avec les objets. Gwenda Hickling constate :

"C'est la vie des Aborigènes, tout nous a été enlevé. C'est une incidence unique qu' on peut vraiment qualifier d'historique. Ça remonte au $\mathrm{XIX}^{\mathrm{e}}$ siècle. Nous avons tout perdu suite à une invasion et que sais-je encore. " (Radio Netherlands Worldwide, 2009)

\section{La rencontre avec les « choses»}

Contrairement à d'autres nations aborigènes, les Bundjalung n'ont ni leur propre centre culturel ni leur musée (Hickling, com. pers., 29/09/2009). Voilà pourquoi il est difficile pour les Bundjalung d'avoir accès à leur culture matérielle et objets d'art. La plupart des objets historiques de la région de la Richmond River se trouvent dans des musées européens tels que le British Museum à Londres, le Hancock Museum (maintenant Tyne \& Wear Archives and Museum) à Newcastle-upon-Tyne, le World Museum à Liverpool, le Pitt Rivers Museum à Oxford, le musée aux Royal Botanic Gardens à Kew et le Rijksmuseum Volkenkunde à Leyde (McBryde, 1978 : 139). Les collections d'Océanie du RMv dont celle concernant les Bundjalung sont entreposées aux réserves externes à quelques quarante minutes en voiture du musée. Lorsque Gwenda Hickling et John Morrissey sont arrivés sur place, ils ne savaient pas trop ce qu'ils devaient en attendre mais ils étaient incontestablement prêts à vivre l'expérience. Avant d'entrer dans l'entrepôt, les deux anciens ont demandé si les objets qu'ils verraient seraient également rapatriés. Cette question essentielle sera reprise à la fin de cet article. En fait, les paroles de Paul
Tapsell sont applicables à la visite des anciens Bundjalung :

«lorsque des anciens de communautés autochtones visitent des musées, ils éprouvent généralement de l'émerveillement, du respect, et se sentent privilégiés d'avoir bénéficié de l'occasion d'entrer en dialogue avec leurs ancêtres dans un voyage au-delà des horizons de la communauté coutumière. »(Tapsell, 2003 : 245)

Leur visite était un cas de "travail de terrain sens dessus-dessous" comme l'explique Ann Fienup-Riordan (2003 : 39) à propos de la visite d'anciens Yup'ik (d'Alaska) à l'Ethnologisches Museum de Berlin. Elle écrit :

«Alors que des anthropologues sont connus pour leurs voyages en terres éloignées en vue d'y étudier les résidents autochtones, dans ce cas-ci, des anciens parmi les autochtones ont voyagé vers le foyer d'anthropologie - le musée - pour y faire leur travail de terrain personnel, arrivant à leurs propres conclusions quant à la valeur des collections ethnographiques qu'ils viennent d'explorer. » (Fienup-Riordan, 2003 : 39)

Nous avons regardé les trente-sept objets de la Richmond River, dont les boomerangs, les boucliers, les massues, les bâtons à fouir, les lances, les filets de pêche et les sacs en vannerie. Alors qu'Isabel McBryde (1978 : 139-187) commente abondamment les collections de la Richmond River du RMv, elle a apparemment manqué la série 295 qui est arrivée en même temps que des ossements humains et qui pour cette raison est au centre de cet article. Elle n'explique pas pourquoi elle n'examine - et avec des commentaires très succincts - que certaines des collections de A. P. Goodwin de date ultérieure. Une comparaison entre la première série d'objets collectés par Goodwin et ses collections ultérieures aurait pu contribuer à mieux préparer la visite des anciens. Pour les deux Aborigènes, c'était la première occasion de voir ces objets puisque rien n'avait été publié à leur propos. Ils leur ont été présentés, triés par type, selon leur agencement dans les réserves du RMv. Douze boomerangs, quatre boucliers, deux massues, cinq lances, trois filets de pêche, deux bâtons à fouir et neuf sacs en vannerie étaient déposés sur une table. Les objets auraient pu être divisés en groupes : des ustensiles avant tout utilisés par les hommes pour se battre, chasser et pêcher - boomerangs, boucliers, massues et lances - et des objets employés par les femmes tels les bâtons à fouir, ou fabriqués par elles tels les sacs en vannerie. Toutefois, la répartition du travail (les femmes rassemblant de la nourriture, parfois du miel, et préparant à manger; les hommes chassant et pêchant) n'était pas rigide comme en témoigne Sharon Sullivan (1978 : 108). Les femmes capturaient également de petits animaux et des reptiles s'ils se trouvaient sur leur chemin pendant 


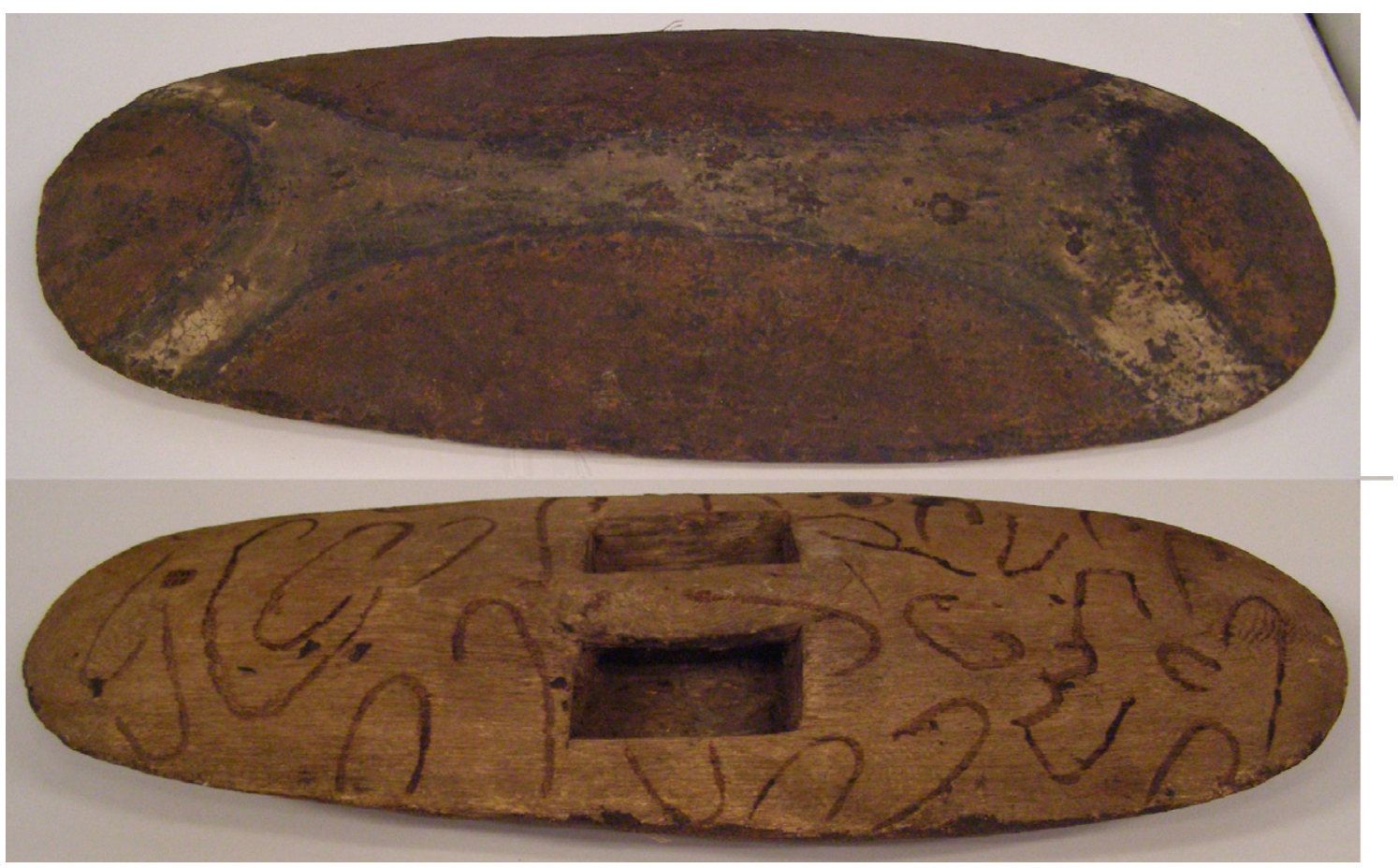

Рното 2. - Bouclier (RMv 295-51), photographié lors de la visite de Gwenda Hickling et John Morrissey.

(C) Fanny Wonu Veys, 30 septembre 2009, 's Gravenzande)

la journée. En général les hommes et les femmes coopéraient dans certaines activités de chasse et de cueillette (Sullivan, 1978 : 108). Il s'agissait donc de ne pas imposer aux anciens Bundjalung une disposition particulière mais de les laisser libres dans leur appréhension des objets.

Avant de relater plus amplement la visite des deux anciens Bundjalung aux réserves externes du RMV, je tiens à faire la description des objets mentionnés ci-dessus. Aucun des boomerangs n'est décoré. Tous, sauf un d'une forme plutốt angulaire, montrent une légère courbe (RMV 29539). Dans cette région, les boomerangs étaient utilisés pour la chasse et la guerre (McBryde, 1978 : 180). Les quatre boucliers sont de plan convexe en coupe transversale. Leur poignée est creusée dans l'épaisseur du bois. Ces boucliers sont en bois très léger. Ils sont peints et caractéristiques de la région de la Nouvelle-Galles du Sud entre la rivière Macleay et les rivières Richmond et Tweed. La provenance géographique est d'autant plus sûre que ce type de bouclier differe très distinctement de celui provenant de la côte de la Nouvelle-Galles du Sud dans la région allant vers le sud de Port Macquarie au Port Jackson. Il s'agit alors d'un bouclier ovale qui présente une coupe transversale en U. Sa poignée, en bois de vigne ou un autre bois souple [sic], est insérée dans deux trous creusés dans l'épaisseur du bois (McBryde, 1978 : 178). Un seul bouclier n'est pas peint (RMV 295-49); un autre est peint en noir et rouge (RMv 295-48). Deux boucliers, l'un peint en noir (RMV 295-50) et l'autre en blanc, noir et rouge (RMV 295-51), sont décorés sur la poignée, d'une part de points gravés et, d'autre part, de stries courbes (photo 2).

Les cinq lances (RMV 295-56 [2 lances] et RMV 295-57 [3 lances]) collectées par Goodwin sont unies et sans barbillons. McBryde (1978: 179) écrit que ce type est le seul observé dans le district de la Richmond River. Cette lance était aussi utilisée pour attraper du poisson même si on préférait les filets. Trois de ces filets ont été collectés par Goodwin (RMV 295-59, RMV 29560, RMV 295-61). Il est intéressant de constater que la lance multi-pointes trouvée le long de la côte est de la Nouvelle-Galles du Sud à partir du district de Sydney en direction du nord vers Hastings et la rivière Macleay et plus vers le nord sur la côte du Queensland, n'était pas utilisée par la population de la région de la rivière Richmond (McBryde, 1978: 179). Les deux massues (RMV 295-54 et RMv 295-55) de la collection sont caractéristiques des massues de la fin du XIx ${ }^{e}$ siècle de l'est de l'Australie. Elles ont une tête cloutée mais pour le reste, elles sont unies. McBryde (1978: 180) prétend que des écrits historiques sembleraient suggérer que la population de la Richmond River connaissait un certain nombre de types de massues et de bâtons à lancer, chacun avec une fonction et un nom spécifiques. Deux bâtons à fouir (RMv 295-52 et RMv 295-53) étaient initialement décrits comme des "massues ". Les femmes les utilisaient pour déterrer les racines de plantes. Il y a deux types de sacs dans les neuf exemplaires: des sacs en 
vannerie spiralée fabriqués à partir d'herbes (RMV 295-62, RMV 295-63, RMV 295-64, RMV 295-65, RMV 295-68, RMV 295-70, RMV 295-71) et des sacs en vannerie en fibres d'écorce (RMV 29567, RMv 295-69). Les deux types de sacs étaient confectionnés par les femmes avec un savoirfaire considérable, mais utilisés par les hommes et les femmes pour de petits objets ou respectivement pour transporter des ustensiles personnels ou des objets précieux. McBryde (1978: 183) affirme que la technique de vannerie nouée était prévalente dans beaucoup de régions d'Australie, tandis que la technique de vannerie spiralée pour faire des sacs et des paniers était confinée à l'est de l'Australie. On constate la présence des colons dans les objets à cause de l'incorporation de matériaux européens. Par exemple, un peu de laine rouge a été insérée dans l'un des sacs (RMV 295-65) et deux sacs ont une lanière en coton (RMV 295-70, RMV 295-71).

La politique générale du musée exige que le personnel et les visiteurs utilisent des gants pour manier les objets. Toutefois il a été expliqué aux deux Bundjalung que le RMv était prêt à s'adapter à leurs suggestions. La réaction lors de la rencontre avec les objets était pleine de retenue. Ils ont porté les gants. Après un début assez silencieux, les langues se sont déliées et ils ont commencé à se souvenir d'histoires racontées par leurs grands-parents. Selon eux, beaucoup d'objets sont encore dans des grottes, mais personne n'ose s'en approcher de peur de déranger les ancêtres. Gwenda Hickling et John Morrissey accordaient la même attention aux objets faits ou employés par des hommes ou des femmes, ne faisant donc aucune différence entre les deux. Aucun des noms vernaculaires n'était connu. Les massues cloutées étaient désignées par le mot australien bundi. L'Australian National Dictionary donne d'autres orthographes telles boondie, bundi, bundy et définit le mot comme "A heavy Aboriginal club" (" une lourde massue aborigène »). Le dictionnaire suggère que le mot est originaire des langues Wiradhuri et Kamilaroi parlées dans la Nouvelle-Galles du Sud.

Les visiteurs ont précisé que les boomerangs n'étaient pas seulement utilisés pour chasser mais aussi pour infliger des coupures lors des deuils. Pourtant, la plupart des histoires ne se rapportaient pas directement aux objets. Ces derniers ne formaient qu'un prétexte à d'autres histoires. Les anciens se souvenaient par exemple qu'ils ne pouvaient pas parler leur langue à l'école. Ils racontaient que quand leurs parents et grandsparents bravaient les interdits et essayaient de parler leur langue maternelle et de transmettre ce qu'ils savaient, ils le faisaient avec une extrême prudence car ils risquaient d'être punis. Les deux anciens ont tenu à dire qu'ils étaient chrétiens maintenant mais que pour autant ils ne voulaient pas oublier le passé. Ils étaient convaincus que le passé, le présent et le futur étaient liés. Ils étaient heureux de savoir que les objets qui jadis avaient appartenu à leurs ancêtres étaient entourés de tant de soins. Jamais auparavant ils ne s'étaient rendu compte qu'ils pouvaient demander l'accès aux objets. À leur retour, ils avaient l'intention de raconter à leurs petits-enfants que ces objets leur étaient accessibles et en voie de préservation pour les futurs petits-enfants. L'éducation, le logement, la santé, l'emploi et le développement du capital étant des questions plus urgentes, le musée est considéré comme un lieu dépositaire approprié dans lequel les objets des ancêtres sont à l'abri, reçoivent le respect qui leur est dû et les soins qui s'imposent. Les objets ancestraux étant en sécurité, accessibles, dans des mains à la fois expertes et attentionnées, le désir de rapatriement n'est plus une priorité absolue et parfois même plus du tout un débat central.

\section{Dans le présent, les objets du passé façonnent le futur}

L'histoire entourant ces objets résume les pratiques de collecte de la fin du XIX ${ }^{e}$ siècle. Mais il y a plus. Ces objets servent de médiateurs, ce qui implique qu'ils transforment le monde social dans lequel ils se trouvent. À partir de la rencontre des anciens Bundjalung avec ces "choses" matérielles, un discours, des commentaires ont jailli chez eux et la volonté de changer la situation qui prévaut actuellement dans la communauté bundjalung s'est déclenchée. Ces objets ont engendré la socialisation des deux anciens qui ont reçu une place plus valorisée dans leur communauté et ont gagné le respect et l'écoute des leurs. L'expérience des objets, en agissant comme ascenseur social, a donc contribué à la restructuration de la vie sociale du groupe. Mais il y a plus. Les " choses " aborigènes ont joué un rôle essentiel dans le développement des relations entre les anciens Bundjalung, le personnel de l'ambassade et celui du musée 7 . Les objets qui nous occupent ont quant à eux reçu un nouveau contexte et ont donc changé. Mais il ne s'agit pas ici d'une relation passive : les objets changent aussi ceux qui les manient. La rencontre avec les objets renfermait des occasions d'influer sur l'avenir et la possibilité aux descendants de John Morrissey et Gwenda Hickling d'accroître leur prestige. Mais la façon dont les objets étaient liés à des 
souvenirs est sans doute d'une plus grande importance. La rencontre avec ces objets ancestraux a fait remonter à la surface une foule de souvenirs, non pas qu'ils aient fait fonction de moyens mnémotechniques; ils paraissaient plutôt aider à structurer la mémoire du fait qu'une histoire particulière n'était pas liée à un objet spécifique, mais que la présence des objets servait de prétexte pour rassembler les bribes de mémoire afin d'en faire une histoire cohérente. L'idée que « les objets sont ce qu'ils sont devenus et non pas ce pour quoi ils ont été faits " (Thomas, 1991:4) prend ici toute sa valeur. Pour les Bundjalung, la rencontre a fait que « les choses " - leurs aspects matériels et immatériels - ne pouvaient plus être oubliées. La préservation de ces objets équivalait presque à la sauvegarde de la mémoire. Dans un sens, c'est l'activité de base des musées. Les objets sont préservés afin de ne pas oublier les histoires auxquelles ils sont liés. Cela permet au personnel du musée de les montrer à d'autres personnes aujourd'hui et de conserver ces objets pour les générations futures. Charles Saumarez Smith (2010 : 550) l'exprime de la façon suivante :

"L'impulsion originelle d'un musée est contenue dans l'idée qu'il représente la cargaison du passé en consignation pour le futur et que nous, dans le présent, devons être témoins de ces choses qui d'une certaine manière sont précieuses ou spéciales ou belles ou éclairantes. »

Tout comme le RMv a joué un rôle de médiateur dans le rapatriement des restes humains aborigènes, tous les musées ont un rôle important de médiateur dans les relations entre communautés, gouvernements et les publics auxquels ils s'adressent. Voilà pourquoi le musée peut toujours être considéré comme le décrivaient Laurier Turgeon et Elise Dubuc (2002: 8) comme une zone de contact entre l'espace extérieur, celui du travail de terrain ethnographique et l'espace interne du musée, une institution de savoir ethnologique. Le dialogue et la pluralité des "voix » sont donc permis dans ce monde en pleine globalisation.

\section{Remerciements}

Je tiens à remercier tout spécialement Gwenda Hickling et John Morrissey pour la confiance qu'ils ont accordée au RMV, au LUMC et à moimême. Leur histoire m’a touchée profondément. Mike Byers, représentant du gouvernement australien, m'a beaucoup aidée en me tenant au courant du déroulement des événements qui ont précédé et suivi le rapatriement des restes humains. Les remarques et les questions de Robert Garbutt de la Southern Cross University lors de la première ébauche de cet article ont indé- niablement fait progresser le développement de mes idées. Merci! Je suis vraiment reconnaissante à Isabelle Leblic, Philippe Peltier et aux lecteurs anonymes d'avoir enrichi mon texte de remarques tout à fait pertinentes dont j'ai tenu compte. Finalement, un grand merci est dû à Françoise Veys, ma mère et Marie-Thérèse Vandendorpe, ma grand-mère, qui ont travaillé sur le texte français.

\section{BIBLIOGRAPHIE}

AtKinson Henry, 2010. The meanings and values of repatriation, in $\mathrm{P}$. Turnbull and M. Pickering (eds), The long way home. The meaning and values of repatriation, New York, Oxford, Berghahn Books, pp. 15-26.

Bolton Lissant, 2003. The object in view. Aborigines, Melanesians, and museums, in $\mathrm{L}$. Peers and A.K. Brown (eds), Museums and Source Communities: A Routledge Reader, London and New York, Routledge, pp. 42-54.

Bonnot Thierry, 2002. La vie des objets. D'ustensiles banals à objets de collection, Paris, Éditions de la maison des sciences de l'homme, coll. Ethnologie de la France 22.

Buchanan Amanda, 2010 (27/09). Remains Buried after 200 Years, The Northern Star.

Byers Mike, 2010 (17/06). RE: Bandjalung repatriation of human remains (Email).

—, 2013 (14/01). RE: Bandjalung repatriation of human remains (Email).

Clifford James, 1988. The Predicament of Culture: Twentieth-Century Ethnography, Literature, and Art, Cambridge, Harvard University Press.

Cubillo Franchesca, 2010. Repatriating our ancestors: who will speak for the dead?, in P. Turnbull and M. Pickering (eds), The long way home. The meaning and values of repatriation, New York, Oxford, Berghahn Books, pp. 20-26.

DAley Louise Tiffany, 1966. Men and a river: Richmond River district 1828-1895, Carlton (Vic.), Melbourne University Press.

Dorsey George A., 1896. The photograph and skeleton of a Native Australian, Bulletin of the Essex Institute 28 (7-12), pp. 57-69.

Duff M.E. Grant, 1890. Report of the evening meetings, Session 1889-1890, Proceedings of the Royal Geographical Society and Monthly Record of Geography (New Monthly Series) $12(1)$, p. 42. 
Goodwin Adolph Peter, 1890. Notes on the Paradise-birds of British New Guinea, Ibis International Journal of Avian Science 32 (2), pp. 150-156.

—, 1896 (20/06). An unknown craft. Canoe washed ashore at Byron Bay, The Northern Star, Lismore (http://trove.nla.gov.au/ndp/ $\mathrm{del} /$ article $/ 72569505$ ?search Term=Descripti on Byron Bay lismore 1896\&searchLimits=, consulté le 6 mars 2013)

Greenfield Jeanette, 2001. The Return of Cultural Treasures, Cambridge, Cambridge University Press.

Hallgren Claes, 2010. Eric Mjöberg and the rhetorics of human remains, in P. Turnbull and M. Pickering (eds), The long way home. The meaning and values of repatriation, New York, Oxford, Berghahn Books, pp. 135-143.

Horton David R., 1994. Bundjalung, in D. Horton (ed.), The Encyclopaedia of Aboriginal Australia. Aboriginal and Torres Strait Islander History, Society and Culture, Canberra, Aboriginal Studies Press for the Australian Institute of Aboriginal and Torres Strait Islander Studies, pp. 163-164.

Hoskins Janet Alison, 1998. Biographical Objects: How Things Tell the Stories of People's Lives, London, UK, New York, NY, Routledge.

Kaplan Flora Edouwaye S., 2011. Making and Remaking National Identities, in S. MacDonald (ed.), A Companion to Museum Studies, Chichester, West Sussex, Wiley-Blackwell, pp. 152-169.

Kopytoff Igor, 1986. The Cultural Biography of Things: Commoditization as Process, in A. Appadurai (ed.), The Social Life of Things, Cambridge, Cambridge University Press, pp. 64-91.

McBryde Isabel, 1978. Museum collections from the Richmond River District, in I. McBryde (ed.), Records of times past: Ethnohistorical essays on the culture and ecology of the New England tribes, Canberra, Australian Institute of Aboriginal Studies, pp. 135-210.

Morton John, 2010. Consigned to Oblivion : People and Things Forgotten in the Creation of Australia, in P. Turnbull and M. Pickering (eds), The Long Way Home. The Meaning and Values of Repatriation, New York, Oxford, Berghahn Books, pp. 96-113.

National Museum Australia, 2008-2009. Repatriation of remains and secret/sacred objects, in National Museum Australia, Annual Report 2008-2009 (http://www.nma.gov. au/about_us/nma_corporate_documents/ annual_report/08_09/part_two/output_ group_1_1_coll_dev_management/repatriation_of_remains_and_secretsacred_objects, consulté le 9 janvier 2013).

Nicks Trudy, 2003. Introduction, in L. Peers and A.K. Brown (eds), Museums and Source Communities: A Routledge Reader, London and New York, Routledge, pp. 19-27.

RADIO NETHERLANDS WORLDWIDE, 2009. Interview with elder Gwen Hickling, (http:// www.rnw.nl/english/article/aboriginal-remains-return-their-homeland, consulté le 9 janvier 2013).

Rosoff Nancy B., 2003. Integrating Native views into museum procedures: hope and practice at the National Museum of the American Indian, in L. Peers and A.K. Brown (eds), Museums and Source Communities: A Routledge Reader, London and New York, Routledge, pp. 72-79.

Saumarez Smith Charles, 2010. The Future of the Museum, in P. Turnbull and M. Pickering (eds), The Long Way Home. The Meaning and Values of Repatriation, New York, Oxford, Berghahn Books, pp. 543-554.

Schmeltz J.D.E., 1899. Jürgen Heinrich Witt, Verein für Naturwissenschaftliche Unterhaltung zu Hamburg 10, pp. 121-124.

Sullivan Sharon, 1978. Aboriginal diet and food gathering methods in the Richmond and Tweed River valleys as seen in early settler records, in I. McBryde (ed.), Records of times past: Ethnohistorical essays on the culture and ecology of the New England tribes, Canberra, Australian Institute of Aboriginal Studies, pp. 101-115.

Tapsell Paul, 2003. Afterworld: Beyond the frame, in L. Peers and A.K. Brown (eds), $M u$ seums and Source Communities: A Routledge Reader, London and New York, Routledge, pp. 242-251.

Thatcher Ulrich Laurel, 2001. The Age of Homespun: Objects and Stories in the Making of an American Myth, New York, Alfred A. Knopf.

The Australian National Dictionary, 2008. Bondi, Oxford, Oxford University Press (http://australiannationaldictionary.com.au/ index.php, consulté le 9 janvier 2013).

THE DAILY NEWs (Perth, WA), 1895 (16/04). Auctions (http://trove;nla;gov;au/ndp/del/ article $/ 78358331$ ?searchterm $=$ Lake $\% 20 \mathrm{Har}-$ dinge\%20goodwin\%201895\&searchLimits, consulté le 9 janvier 2013). 
THE NORTHERN STAR (Lismore, NSW), 1887 (12/01). Commercial (http://trove.nla.gov. $\mathrm{au} / \mathrm{ndp} / \mathrm{del} /$ article/71721014?searchTerm $=\mathrm{Z}$ adoc\%20Goodwin\&searchLimits=, consulté le 9 janvier 2013).

—, 1914 (21/09). Death of Mr. A.P. Goodwin, The Northern Star, Lismore, NSW (http://trove.nla.gov.au/ndp/del/article/72 141917 ? search Term =adolph\%20Peter $\% 20$ goodwin\&searchLimits=, consulté le 9 janvier 2013).

The West Australian (Perth, WA), 1895 (13/04). Summary of Auction Sales (http://trove.nla.gov.au/ndp/del/ article/4548466? search Term=Lake\%20Hardinge $\% 20$ goodwin $\% 201895 \&$ searchLimits=, consulté le 9 janvier 2013).

Thomas Nicholas, 1991. Entangled Objects. Exchange, Material Culture, and Colonialism in the Pacific, Cambridge, Massachusetts - London, Harvard University Press.

Turgeon Laurier et Élise Dubuc, 2002. Musées d'ethnologie : nouveaux défis, nouveaux terrains, Ethnologies 24 (2), pp. 5-18.
Turgeon Laurier (ed.), 2004. L'appropriation et la désappropriation des objets, Revue d'histoire de la culture matérielle 59, Ottawa, Ontario, Canada Science and Technology Museum, Musée des sciences et de la technologie du Canada 2005.

Turgeon Laurier, 2007. La mémoire de la culture matérielle et la culture matérielle de la mémoire, in O. Debary and L. Turgeon (éds), Objets \& Mémoires, Paris-Québec, Éditions de la maison des sciences de l'homme - Les Presses de l'Université Laval, pp. 16-36.

Turnbull Paul, 1997. Ancestors, not Specimens: Reflections on the Controversy over the Remains of Aboriginal People in European Scientific Collections, The Electronic Journal of New Zealand and Australian History (http://www.jcu.edu.au/aff/history/articles/ turnbull.htm, consulté le 9 janvier 2013).

Turnbull Paul, 2010. The Vermillion Accord and the Significance of the History of the Scientific Procurement and Use of Indigenous Australian Bodily Remains, in P. Turnbull and M. Pickering (eds), The Long Way Home. The Meaning and Values of Repatriation, New York, Oxford, Berghahn Books, pp. 117-134. 


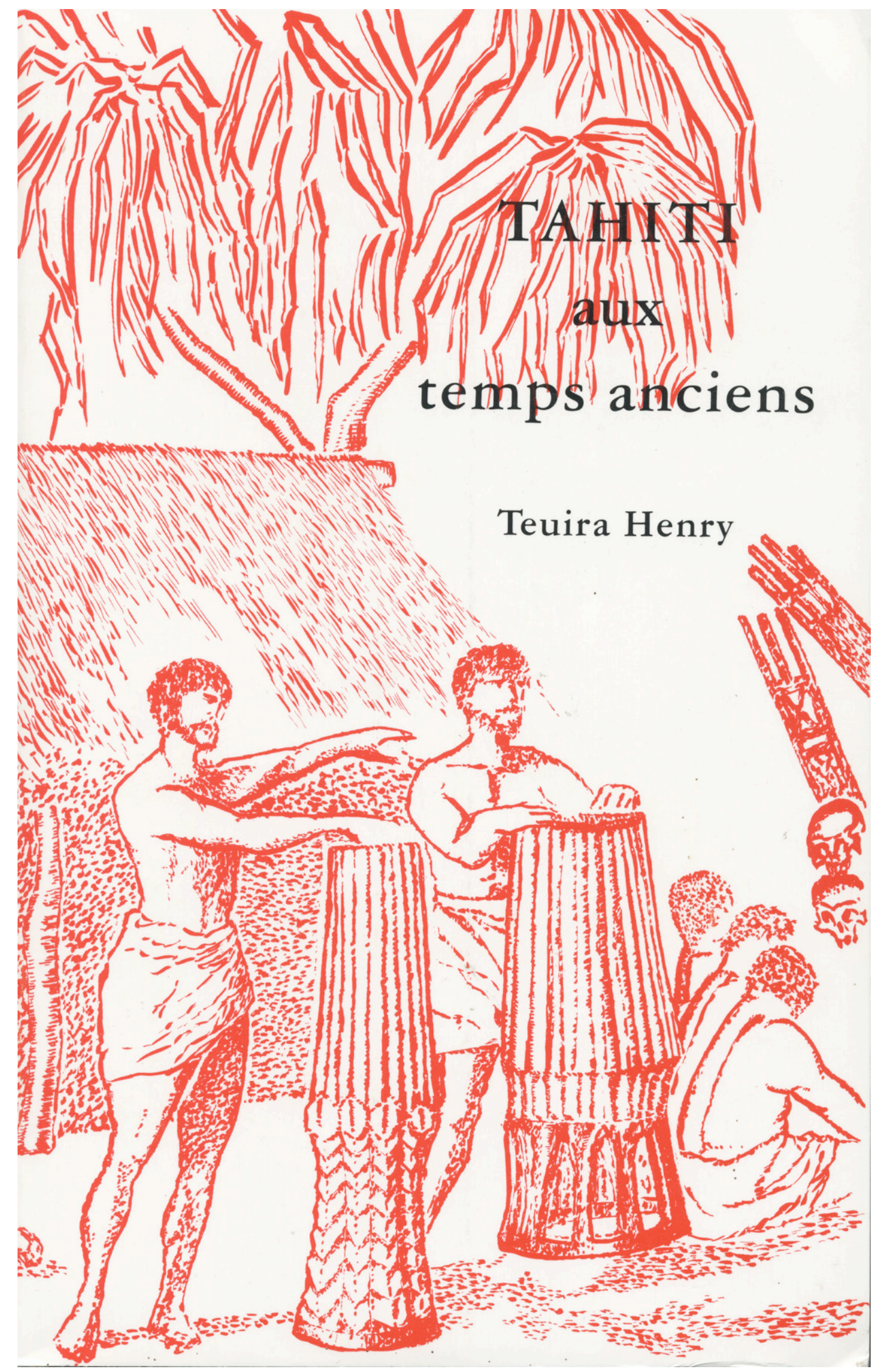

Nouvelle édition - $31 €$ avec paiement en ligne

En vente sur http://oceanistes.org/oceanie/ spip.php?article189 